\title{
Autodescripciones sexuales y estereotipos de género en estudiantes terciarios y universitarios
}

Mercedes Fernández Liporace *

\section{Resumen}

Se presentan resultados parciales de un estudio cuyo objetivo es determinar la manera en la que una muestra intencional de 248 estudiantes terciarios y universitarios residentes en la ciudad de Buenos Aires y el conurbano bonaerense se autodescriben en relación a sus comportamientos sexuales: se verifican diferencias significativas entre ambos sexos en determinados adjetivos vinculados a los roles más tradicionalmente asignados a hombres y mujeres en las relaciones sexuales. Se efectúa, además, una comparación con los hallazgos reportados en una investigación llevada a cabo por Williams \& Best (1994) acerca de estereotipos de género. Los instrumentos utilizados fueron una encuesta sociodemográfica y un listado de 70 adjetivos que aluden a descripciones sexuales.

Palabras claves: Sexual. Autodescripción. Estudiantes universitarios.

\section{SEXUAL SELF- DESCRIPTIONS AND GENDER STEREOTYPES IN COLLEGE STUDENTS}

\section{Abstract}

The paper presents partial results from a study carried out to analyze the way young college students self-describe their sexual behavior in relation with sex. Data show significant differences in certain adjectives, linked with traditional roles assigned to women and men in sexual relationships; an additional comparison with results about gender sterotypes reported by Williams \& Best (1994) is made. A survey on sociodemographic data and a list of 70 adjectives that describe sexual behaviors were administered to a sample of 248 college students from Buenos Aires city and its suburban area.

Key words: Sexual. Self-descriptions. College students.

\footnotetext{
* Profesora adjunta Facultad de Psicología, Universidad de Buenos Aires. Becaria posdoctoral CONICET. Independencia 3065 (1225) Buenos Aires. Argentina E-mail: mliporac@psi.uba.ar
} 


\section{Introducción}

Es un supuesto asumido en la Psicometría y la Evaluación Psicológica que las autodescripciones se relacionan con el autoconcepto; así, el sí mismo o self puede estudiarse en su vinculación con los distintos roles sociales que un sujeto asume en su interacción con otros y que condicionan sus relaciones interpersonales.

Clásicamente, James (1890) se refiere al Yo en tanto agente de conocimiento, atribuyéndole continuidad, diferenciación y volición como características descriptivas básicas que construyen y mantienen el sentido de identidad. En este modelo, la noción de sí mismo o self se hace extensiva al contexto - contrastando con la postura dualística cartesiana que sostiene una marcada división entre cuerpo y mente -, así como entre persona y contexto, admitiéndose la existencia de varios o diferentes sí mismos; cada persona posee tantos Yo como sujetos existan que lo reconozcan como una individualidad: la identidad humana es única y a la vez, múltiple. El Yo, como proveedor continuo y permanente información, permite la formación de la identidad personal, posibilitando que el individuo se sienta uno mismo en distintos momentos del ciclo vital, así como distinto los demás; esta postura sostiene la existencia de un Yo empírico constituido por todo aquello que se reconoce como propio.

Basándose en la obra de James y en la del novelista ruso Bakhtin (1973), Hermans, Kempen \& Van Loon (1992) caracterizan la identidad según el concepto de multiplicidad dinámica de diferentes posiciones del Yo: este último es capaz de desplazarse desde una posición espacial hacia otra en virtud de cambios temporales o situacionales, posibilidad que supone la asunción de distintos roles. Los autores preconizan la superación de posturas individualistas y racionalistas, sugiriendo la noción de self dialógico: el Yo individual posicionándose de distintas maneras en el tiempo y en el espacio; ubicado en alguna de ellas, está capacitado para comprender, criticar o contradecir al posicionado en espacios y tiempos diferentes. Este sí mismo dialógico es fundamentalmente social, a partir de conceptualizar que los otros - no necesariamente reales - , por medio de sus múltiples voces, forman parte de cada subjetividad individual.

Factores sociales y culturales integran el espacio personal subjetivo asumiendo tres formas: a) ideas y formas de pensamiento, b) códigos internalizados referidos a las expresiones aceptadas, permitidas, y c) modos de concreción de la dis-

\footnotetext{
* Se agradece la colaboración de jóvenes graduados y estudiantes de la carrera de Psicología de la Universidad de Buenos Aires, participantes en la recogida y entrada de datos, así como en el relevamiento bibliográfico: Alicia Barreiro, Eusebio Benegas, Florencia Donati, Josefina Landgraf, Lucila López, Ma. Laura Lupano, Paula Ongarato, Lorena Puchetti, Ma. Laura Trol y Lucia Vinciguerra. Estos resultados corresponden al del PROYECTO UBACYT: P043, EMOCIONES Y SALUD MENTAL: IMPACTOS SALUGÉNICOS Y PATOGÉNICOS DE LAS RELACIONES ROMÁNTICAS EN ADOLESCENTES Y JÓVENES. 2001-2002, dirigido por María M. Casullo.
} 
tribución de esas ideas y códigos en el colectivo social. Resulta importante resaltar el rol que en nuestros días desempeñan los medios masivos de comunicación - TV, radio, Internet - en tanto transmisores de mensajes y significados alusivos a los comportamientos sexuales supuestamente aceptados y deseables.

Muchos de los roles asumidos por el Yo en el proceso de evolutivo - ser hijo/a, alumno/a, hermano/a, vecino/a, padre o madre, entre otros, y que determinan la estructuración de la identidad, se hallan vinculados con ideas, pautas, mensajes y códigos referidos al género y al ejercicio de la sexualidad. Lo femenino y lo masculino se construyen dialógicamente y en relación con los comportamientos esperados, aprobados o cuestionados. La femineidad se estructura en relación y por oposición a lo definido como masculino, y así se van perfilando semejanzas y diferencias.

Hermans (2001) caracteriza la conformación de identidades contextuadas en los acelerados procesos de globalización y tecnificación que se dan en la actualidad. Sostiene que el sí mismo o self se compone de una enorme cantidad de posiciones, resultantes de numerosas interrelaciones entre manifestaciones culturales diversas. Por otra parte, tales posiciones del Yo son relativamente heterogéneas: grupos que históricamente funcionaron de manera cerrada y homogénea pueden luego formar parte de un sistema de interconexión social más amplio. Por otra parte, se producen abruptas alteraciones en las ofertas disponibles: adelgazamiento rápido y drástico, conservación de una eterna juventud, transplante de órganos, fertilidad asistida. Los mensajes transmiten como deseable lo que no necesariamente se es capaz de lograr u obtener, dado que no se cuenta con los recursos o valores requeridos.

Desde otro enfoque, la forma en la que los individuos autodescriben su sexualidad resulta coherente con las representaciones sociales que comparten sobre los comportamientos esperables para varones y mujeres con respecto al desempeño de los roles sexuales: estos significados sirven a modo de sistemas de referencia que posibilitan la interpretación y clasificación de circunstancias, fenómenos o personas, entre otros elementos (Jodelet, 1986). Las representaciones que cada persona sostiene en cuanto al desempeño sexual según género se relacionan con las normativas culturales que establecen los atributos que deben tener varones y mujeres respecto de su subjetividad y de los roles a asumir (Meler, 1994).

Existen tres forman diferentes, aunque relacionadas, de ver a mujeres y varones: las autopercepciones, la ideología de rol de género y los estereotipos de género. Estos últimos son un tipo especial de teoría implícita de la personalidad que se relaciona con las características que son atribuidas a uno ó más grupos sociales completos, en este caso, las mujeres y los varones. Las autopercepciones se definen como el grado en que los estereotipos de género se incorporan a los autoconceptos de cada sexo, en tanto que la ideología de rol del género es el conjunto de creen- 
cias sobre las relaciones de rol que se consideran adecuadas entre varones y mujeres (Wiilliams y Best, 1994). En las décadas de los 80 y los 90, estos autores desarrollaron una investigación con niños, adolescentes y estudiantes universitarios de más de 30 países de los cinco continentes. En ella se instaba a los sujetos a seleccionar de un listados de 300 adjetivos, aquéllos más comúnmente asociados con las mujeres por una lado, con los varones por el otro, y con ambos sexos por igual en último término. Se verificó un altísimo acuerdo entre todos los grupos estudiados, mostrándose así que los estereotipos de género observan una alto grado de semejanza, aunque con variaciones culturales sistemáticas: por caso, en naciones protestantes los estereotipos poseían mayor diferenciación que la que se registraba en países católicos; a la vez, la estereotipia sexual parece rigidizarse durante la adolescencia y la adultez temprana. A medida que la edad infantil deja paso a la adolescencia, la diversidad intercultural en las respuestas disminuía: esto permite suponer que los estereotipos adquieren más semejanza con el aumento de la edad.

La investigación acerca de las diferencias sexuales se basa en el supuesto de que existe una diferencia biológica irreductible entre los sexos; el sexo no es una variable unidimensional, se distinguen en él cinco dimensiones que, normalmente correlacionan entre sí en la mayoría de los individuos: ellas son el sexo cromosómico, el sexo gonadal, el hormonal, el sexo de los órganos accesorios internos y la función y apariencia de los genitales externos (Edley y Wetherell, 1995). Maccoby y Jacklin (1974) efectuaron una de las mayores revisiones de la literatura referida al área, comprobando que numerosos estereotipos populares de género no resultan de la evidencia empírica, existiendo una enorme cantidad de mitos, tanto populares como científicos.

Según el enfoque sociobiológico, los roles de género se han formado gradualmente en el curso de la evolución como parte de la adaptación del ser humano a su ambiente; de esta forma, cada sexo estaría equipado con características físicas e instintivas apropiadas para cada una de sus actividades y responsabilidades (Durkin, 1995). Universalmente, los atributos que las mujeres encuentran atractivos en los varones se hallan vinculados con la provisión de recursos y la protección de sí misma y de las crías, en tanto que los varones jerarquizan la belleza física, buscando una potencial madre, la más joven y saludable, capaz de procrear más eficazmente (Buss, 1987, 1994; Buss et al, 1990; Schmitt y Buss, 2001). Así, el macho humano se halla siempre dispuesto a mostrar su habilidad como protector y conquistador, presentando posturas agresivas y de dominio, mientras que la hembra humana se mostrará atractiva y arreglada, pero tímida y haciéndose la difícil, puesto que evaluar la factibilidad y ventaja de esta potencial unión requiere algún tiempo, aunque todas estas evaluaciones y comportamientos se ejecuten de manera no consciente (Archer, 1992).

Para las teorías biológicas, las diferencias genéticas y hormonales son responsables de muchos atributos ligados al sexo: parecería que los niños están biológi- 
camente programados para cierta clase de actividades compatibles con el rol masculino, por lo que estarían predispuestos a la agresión, la asertividad o el juego rudo por caso, en virtud de su físico más musculoso, su más alto nivel de actividad, su mayor demanda, su temperamento más irritable y sus umbrales más altos al dolor. Las niñas, en cambio, serían más dóciles, no demandantes, verbales en grado superlativo, idealmente aptas para asumir roles de cuidadoras, cooperativas y sumisas. Estas predisposiciones resultarían del hecho verificado de que se conciben aproximadamente 120 varones por cada 100 niñas; esta proporción disminuye a 110:100 para fetos supervivientes a término, a 106:100 para nacidos vivos - se abortan espontáneamente más fetos masculinos, más varones nacen muertos o mueren por traumas de nacimiento o malformaciones congénitas -. A lo largo del ciclo vital el varón es más vulnerable a asfixias, parálisis cerebral, convulsiones, infecciones virales, úlceras, cardiopatías y algunos tipos de cáncer. Dado que, además, el cromosoma $\mathrm{Y}$ es más pequeño, el varón es más susceptible a trastornos hereditarios. De ahí su agresividad y actividad exacerbadas, destinadas a preservar su existencia más amenazada (Dorner, 1976; Jost, 1970; Ornstein, 1986).

Por su parte, la teoría biosocial hace hincapié en la manera en que los neonatos influyen con sus temperamentos en la forma en que otros los tratan, contribuyendo, así, a su propio desarrollo: el sexo del infante determina, al igual que su temperamento, la actitud de los otros hacia él; si aquéllos poseen expectativas estereotipadas sobre las diferencias entre los sexos, las expresarán por medio de su interacción con el niño (Rutter y Rutter, 1992). El marbete sexual que se le adjudica, determina el modo en que se socializará al bebé (Money y Ehrhardt, 1972).

Las relaciones entre lo masculino y lo femenino son construidas desde la lógica de la modernidad en términos atributivos, binarios: otorgan al género masculino las propiedades del modelo humano (humanidad = hombre); a partir de él tipifican las características de la mujer, dado que consideran solamente dos términos: masculino y femenino y, por otro lado, transforman uno de los términos en inferior, complemento, suplemento (Bonder, 1985). Esta lógica se mantiene hasta la actualidad mediante distintos mitos circulantes en el Imaginario Social, que han descripto a la mujer como destinada a ser madre, eróticamente pasiva y creyente en el amor romántico, manteniéndola en el ámbito familiar privado y afectivizado, en tanto que el hombre es definido como proveedor económico, activo y que se desempeña en el ámbito público y racionalizado (Fernández, 1993).

Una conceptualización similar de los roles sexuales pertenece a Hofstede (1999) que enumera cuatro dimensiones culturales que aparecen constantes en la totalidad de las organizaciones sociales. Una de ellas es la de Masculinidad Feminidad : lo masculino es interpretado como un atributo psicosocial vinculado a valores tales como el éxito, el dinero y la posesión de bienes y objetos. Lo feme- 
nino se define con aquellos comportamientos en los que predominan la protección y los cuidados del otro, así como el interés por la calidad de vida. "Los temas socioantropológicos relacionados con esta dimensión tienen que ver con la elección y adjudicación de roles sexuales y el efecto que ellas tienen sobre los autoconceptos personales" (Casullo y cols., 1991, p.97).

Para el psicoanálisis clásico, durante la fase pregenital sádico-anal se determina la diferencia que atraviesa la vida sexual, a pesar de que aún no puede pensarse en masculino y femenino, sino en activo y pasivo. Después de atravesar la conflictiva edípica se tipifican los pares sexuales: la mujer accede a la feminidad al reprimir la actividad que se manifestó durante la fase fálica, así como al trocar la zona erógena rectora del clítoris, equivalente del pene, a la vagina. La feminidad se consolida en torno a la pasividad y el masoquismo (Freud, 1905, 1923). Siguiendo a Freud, Laplanche y Pontalis (1981) conciben lo masculino-femenino como el modo de situarse el individuo en asociación con su sexo biológico: la información biológica no resulta suficiente para explicar el comportamiento sexual. Definirse como varón o como mujer implica, además, asumir lo que socialmente es esperable en un hombre y una mujer, no es algo que se adquiere a partir del nacimiento sino que es un camino a recorrer en el que lo masculino y lo femenino son puntos de destino. Ideas semejantes postula Fernández (1993) al aseverar que la construcción de la subjetividad se da inmersa en un proceso sociohistórico.

Siguiendo las afirmaciones de Freud, Lacan (1973) sostiene que lo masculino y lo femenino son posiciones simbólicas: que el individuo se asuma como hombre o como mujer es esencial en la construcción de la subjetividad puesto que el sujeto es fundamentalmente sexuado.

Las autodescripciones sexuales, entonces, pueden ser enfocadas desde distintas perspectivas en virtud de su complejidad: la sociobiológica, la biológica pura, la psicológico - social, el psicoanálisis y los estudios sobre género, entre otros. Sin embargo, resulta importante resaltar que ellas constituyen un área novedosa sobre la que existe una magra cantidad de resultados empíricos publicados.

Actualmente, los dispositivos de socialización de cada sexo se han acercado; no obstante, las separaciones iniciales siguen produciendo fuertes diferencias de comportamiento, orientación y recorrido. El varón continúa representado ejerciendo roles mayoritariamente públicos o instrumentales, en tanto que la mujer permanece vinculada con roles privados, estéticos y afectivos. No resulta posible analizar la identidad sexual sin asociarla al discurso occidental referido al amor y a las relaciones románticas (Lipovetsky, 1999). A pesar de que la cultura amorosa actual proclama la libertad y la igualdad de los sexos, cada sexo posee dife- 
rente significación acerca del amor: para la mujer la necesidad de amar es más constante y más dependiente, en tanto que para el hombre es más un ideal contingente que un motivo exclusivo para vivir (de Beauvior, 1990). Todo ello influye directamente sobre la constitución de la identidad sexual.

En la década de los sesenta surge un movimiento feminista que reacciona ante la forma en que se socializa a las mujeres, sometiéndolas al ideal del amor romántico; así, el acento se desplaza desde los sentimental hacia lo sexual: lo fundamental no será ya amar sin límites sino gozar sin trabas. Sin embargo, Lipovetsky (1999) destaca que las diferencias de género son, en nuestros días, menos exclusivas y más flexibles, aunque la dinámica igualitaria no ha logrado alterar el orden de la diferencia que se ha perpetuado durante siglos.

El influjo de las ideas e instituciones religiosas sobre la construcción de las actitudes y comportamientos sexuales resulta otro punto destacable. Entre 1550 y 1750 la concepción judeo-cristiana acerca del sexo produjo un cambio profundo en los comportamientos asociados con él: aparecieron ideas novedosas acerca del cuerpo, cambios en las características del matrimonio, conceptos nuevos sobre las diferencias de género, mayor importancia simbólica de la sexualidad, así como también nuevos métodos de control de la vida sexual de los individuos. Hasta fines del siglo XVIII los sujetos no consideraban que ellos mismos fueran poseedores de una sexualidad, así como tampoco clasificaban como sexuales algunos elementos que actualmente nos parecen de esta clase de manera evidente. Así, resulta claro que las creencias religiosas pesan fuertemente en la constitución de los comportamientos y las actitudes vinculados a la constitución de la identidad sexual. Para el crsitianimo la sexualidad se moteja como pecaminosa per se, mientras que en el islamismo, no se caracteriza como tal.

Las oposiciones genéricas de la sociedad actual resultan de la destradicionalización de la familia, se dan al interior de la pareja y se recrean en la cama, en la mesa o en la interacción con los hijos. Las ofertas del mercado laboral económicamente globalizado, tanto para mujeres como para hombres, obstaculizan el cumplimiento de las asignaciones de nuevos roles, dado que presuponen el modelo tradicional de familia nuclear con sus características genéricas fundamentales (Beck y Beck-Gernsheim, 2001). En nuestros días se observa que hombres y mujeres conciben amor y compromiso de forma diferente, distinción que determina la conformación de identidades diferentes según género (Townsend, 1998).

\section{Planteo del problema de investigación}

Teniendo en cuenta los desarrollos teóricos y la evidencia empírica que se deta- 
lla en párrafos anteriores, el interés se centra en estudiar la manera en que jóvenes estudiantes terciarios y universitarios describen sus propios comportamientos sexuales, con el objeto de establecer diferencias según sexo y de comparar, por último, estos resultados con los informados por Williams y Best (1994) en su trabajo sobre estereotipos sexuales, realizado en 30 países. Particularmente, focaliza la atención en determinar si los atributos que cada sexo informa para sí en sus comportamientos sexuales coinciden aproximadamente o en un sentido general con los vinculados a los estereotipos sexuales transculturalmente verificados.

\section{Objetivos del estudio}

1. Analizar las autodescripciones sexuales brindadas por estudiantes terciarios y universitarios, residentes en la ciudad de Buenos Aires y el conurbano bonaerense, comparando las diferencias existentes según sexo.

2. Interpretar estos resultados a la luz de los informados en el estudio transcultural sobre estereotipos sexuales (Williams y Best, 1994).

\section{Método}

Tipo de diseño: descriptivo - correlacional.

\section{Hipótesis de trabajo:}

1. Se verificarán diferencias estadísticamente significativas entre los adjetivos seleccionados por varones y mujeres para describir sus propios comportamientos sexuales.

\section{Instrumentos:}

a) Un listado de 70 adjetivos que hacen referencia a comportamientos o características sexuales; cada término debe ser calificado por los participantes sobre la base de una escala tipo Likert con puntuaciones que varían entre la categoría 1 (totalmente inexacto) a la 9 (totalmente exacto), tomando en cuenta en qué medida caracterizan el comportamiento sexual personal de quien responde.

b) Una encuesta sociodemográfica donde se recogen los datos que sirven para describir las características de la muestra analizada. 


\section{Muestra}

Participaron en la investigación 248 estudiantes de 17 a 40 años (edad media $=22.60 ; \mathrm{sd}=3.33$ ) seleccionados en forma intencional y pertenecientes a diferentes institutos terciarios y universidades, públicos y privados, de la ciudad de Buenos Aires y el conurbano bonaerense (55\% de mujeres; $45 \%$ de varones).

El 96\% de los sujetos afirma ser heterosexual; el 60\% responde que tiene actualmente una relación de pareja, en tanto que el $40 \%$ no la tiene. El $50 \%$ de los varones y el $32 \%$ de las mujeres aseveran no ser religiosos; informan una religiosidad moderada el $45 \%$ de los participantes del sexo masculino y el $65 \%$ de sus pares femeninos. Sólo un $5 \%$ y un $3 \%$ de hombres y mujeres declara, respectivamente ser muy religioso.

\section{Análisis de los datos obtenidos}

Dado que se analizan datos ordinales (escala likert de nueve categorías), se trabaja con contrastes no paramétricos, utilizando la prueba de Mann- Whitney con el objeto de determinar la presencia de diferencias significativas según sexo.

\section{Resultados}

Se resume a continuación la frecuencia de respuestas por sexo para los diez vocablos más elegidos - respondidos con las tres categorías de mayor acuerdo con la descripción propuesta (7-8-9: bastante, muy y totalmente exacto) -, (Tabla 1).

Tabla 1: Adjetivos elegidos con mayores frecuencias (categorías de respuesta 7 a 9, mayor acuerdo con la descripción propuesta). Distribución según sexo.

\begin{tabular}{lcc|lcc}
\hline \multicolumn{2}{c|}{ SEXO MASCULINO } & \multicolumn{3}{c}{ SEXO FEMENINO } \\
\hline \multicolumn{1}{c}{ Adjetivo } & N & $\%$ & Adjetivo & N & $\%$ \\
\hline Heterosexual & 106 & 96 & Heterosexual & 130 & 96 \\
Masculino & 101 & 92 & Cariñosa & 122 & 90 \\
Varonil & 100 & 91 & Apasionada & 119 & 87 \\
Cariñoso & 92 & 84 & Monógama & 118 & 87 \\
Tierno & 86 & 78 & Tierna & 118 & 87 \\
Apasionado & 82 & 74 & Fiel & 117 & 86 \\
Gentil & 81 & 74 & Romántica & 110 & 81 \\
Divertido & 80 & 73 & Femenina & 107 & 79 \\
Romántico & 79 & 72 & Divertida & 107 & 79 \\
Espontáneo & 77 & 70 & Espontánea & 105 & 77 \\
\hline
\end{tabular}


Tal como puede observarse, existe un alto nivel de coincidencia: ambos sexos se describen como heterosexuales, cariñosos, apasionados, tiernos, románticos, divertidos y espontáneos. Los varones seleccionan tres términos que no seleccionan las mujeres: GENTIL, VARONIL Y MASCULINO. Por su lado, las mujeres escogen los vocablos MONÓGAMA, FIEL Y FEMENINA, que no son elegidos por el grupo masculino.

La Tabla 2 presenta los términos autodescriptivos en los que se verificaron diferencias significativas según sexo. La primera columna correspondiente a cada sexo - a favor del que se hallaron las diferencias - contiene los adjetivos; la segunda, el resultado valor de $\mathrm{Z}$ para la prueba $\mathrm{U}$ de Mann- Whitney, en tanto que la tercera indica el nivel de significación de la diferencia.

Tabla 2: Adjetivos con diferencias significativas según sexo.

\begin{tabular}{|c|c|c|c|c|c|}
\hline \multicolumn{3}{|c|}{ SEXO MASCULINO } & \multicolumn{3}{|c|}{ SEXO FEMENINO } \\
\hline Adjetivo & $\mathbf{Z}$ & Sig. & Adjetivo & $\mathbf{Z}$ & Sig. \\
\hline Adúltero & -4.34 & $\star *$ & Afeminada & $\begin{array}{l}-10.04 \\
\end{array}$ & $\star \star *$ \\
\hline Descarado & -2.15 & * & Amorosa & -2.87 & ** \\
\hline Desvergonzado & -3.30 & ** & Casadera & -3.56 & ** \\
\hline Escabroso & -2.61 & ** & Compasiva & -2.18 & * \\
\hline Fácil & -3.46 & ** & Coqueta & -4.65 & ** \\
\hline Galante & -3.60 & ** & Femenina & -12.89 & ** \\
\hline Grosero & -2.92 & ** & Fiel & -4.05 & ** \\
\hline Infiel & -3.92 & ** & Inocente & -2.34 & * \\
\hline Inmoral & -2.54 & * & Insinuante & -3.27 & ** \\
\hline Libertino & -3.12 & ** & Monógama & -5.09 & ** \\
\hline Lujurioso & -3.17 & ** & Romántica & -3.17 & ** \\
\hline Masculino & -13.60 & ** & Seductora & -2.23 & * \\
\hline Mujeriego & -9.56 & ** & Sensual & -2.30 & * \\
\hline Ninfómano & -2.62 & ** & & & \\
\hline Obsceno & -4.19 & ** & & & \\
\hline Ordinario & -3.27 & ** & & & \\
\hline Pervertido & -3.66 & ** & & & \\
\hline Polígamo & -5.09 & ** & & & \\
\hline Promiscuo & -6.46 & ** & & & * $p>0.05$ \\
\hline Varonil & -13.62 & ** & & & $* * p>0.01$ \\
\hline
\end{tabular}

Como puede apreciarse a partir de la lectura de la Tabla 2, se hallaron diferencias significativas a favor de las mujeres para los siguientes adjetivos: afeminada, amorosa, casadera, compasiva, coqueta, femenina, fiel, inocente, insinuante, monógama, romántica, seductora y sensual.

Las diferencias a favor de los hombres se registraron para los vocablos adúltero, descarado, desvergonzado, escabroso, fácil, galante, grosero, infiel, inmoral, libertino, lujurioso, masculino, mujeriego, ninfómano, obsceno, ordinario, pervertido, polígamo, promiscuo y varonil.

Resulta interesante destacar que la mayoría de los términos que diferencian a 
ambos sexos, no figuran entre los elegidos con más frecuencia por los participantes en este estudio (v. Tabla 1). Es evidente que las mujeres se consideran más fieles y monógamas que los hombres. La polarización que se advierte es notable: los varones eligen vocablos que remiten a un personaje dado a la conquista, promiscuo, lujurioso; las mujeres, en cambio, se describen como receptivas a la conquista, pero preparándose para tal ocasión, con atributos destinados a resultar agradables a los ojos del otro.

La Tabla 3 compara los resultados obtenidos por Williams y Best (1994) con los informados en este trabajo para el caso de los varones. Las dos columnas de la izquierda corresponden a los términos recabados por aquellos autores como más comúnmente asociados con varones y mujeres en 30 países, en tanto que la de la derecha resulta de esta investigación. Recuérdese que las listas de adjetivos presentadas en ambos estudios es diferente (300 vs. 70 reactivos). Es interesante que ambas descripciones, aunque diferentes en cuanto a las características a las que aluden - en el primer caso, son generales; en el segundo, referidas a comportamientos sexuales -,coinciden en presentar un individuo dominante, activo, agresivo, arrogante, inescrupuloso, descarado, dado al placer y a la conquista, entre otras. Sólo dos atributos - que figuran en negritas - no se corresponden con tales connotaciones: ellos son confiado y flojo.

Tabla 3: Comparación de resultados sobre estereotipos de género y autodescripciones sexuales (varones)

\begin{tabular}{|c|c|c|}
\hline \multicolumn{2}{|c|}{ Estereotipos de género (Williams \& Best, 1994) } & Autodescripciones sexuales \\
\hline Activo & Hostil & Adúltero \\
\hline Agresivo & Imperturbable & Descarado \\
\hline Agudo & Indiferente & Desvergonzado \\
\hline Ambicioso & Individualista & Escabroso \\
\hline Amigo del placer & Inescrupuloso & Fácil \\
\hline Arrogante & Ingenioso & Galante \\
\hline Autocrático & Inquieto & Grosero \\
\hline Aventurero & Insensible & Infiel \\
\hline Capaz & Inventivo & Inmoral \\
\hline Cínico & Ocurrente & Libertino \\
\hline Con amplitud de intereses & Odioso & Lujurioso \\
\hline Con iniciativa & Oportunista & Masculino \\
\hline Confiado & Preciso & Mujeriego \\
\hline Cruel & Presuntuoso & Ninfómano \\
\hline Desordenado & Progresista & Obsceno \\
\hline Determinado & Racional & Ordinario \\
\hline Dominante & Rápido & Pervertido \\
\hline Emprendedor & Realista & Polígamo \\
\hline Engreído & Rígido & Promiscuo \\
\hline Estricto & Rudo & Varonil \\
\hline Firme & Serio & \\
\hline Flojo & Tacaño & \\
\hline Fuerte & Testarudo & \\
\hline Gracioso & Tosco & \\
\hline Gritón & Valiente & \\
\hline
\end{tabular}


Examinando la Tabla 4 puede observarse que los adjetivos recogidos en ambos estudios muestran, mayoritariamente, connotaciones relacionadas con la debilidad, la protección hacia otros, así como cierta actitud inocente y dependiente.

Tabla 4: Comparación de resultados sobre estereotipos de género y autodescripciones sexuales (mujeres).

\begin{tabular}{|c|c|c|}
\hline \multicolumn{2}{|c|}{ Estereotipos de género (Williams \& Best, 1994) } & Autodescripciones sexuales \\
\hline $\begin{array}{l}\text { Afectuosa } \\
\text { Agradable } \\
\text { Agradecida } \\
\text { Amable } \\
\text { Apacible } \\
\text { Apocada } \\
\text { Aprensiva } \\
\text { Autocompasiva } \\
\text { Bondadosa } \\
\text { Cálida } \\
\text { Cauta } \\
\text { Complicada } \\
\text { Comprensiva } \\
\text { Confusa } \\
\text { Criticona } \\
\text { Curiosa } \\
\text { Débil } \\
\text { Dependiente } \\
\text { Emocional } \\
\text { Encantadora } \\
\text { Excitable } \\
\text { Fingida } \\
\text { Frívola } \\
\text { Gentil } \\
\text { Habladora }\end{array}$ & $\begin{array}{l}\text { Imaginativa } \\
\text { Indulgente } \\
\text { Inestable } \\
\text { Melindrosa } \\
\text { Modesta } \\
\text { Nerviosa } \\
\text { Paciente } \\
\text { Poco inteligente } \\
\text { Pudibunda } \\
\text { Quejumbrosa } \\
\text { Quisquillosa } \\
\text { Sensible } \\
\text { Sentimental } \\
\text { Sexi } \\
\text { Sin ambiciones } \\
\text { Sofisticada } \\
\text { Soñadora } \\
\text { Sugestionable } \\
\text { Sumisa } \\
\text { Supersticiosa } \\
\text { Temerosa } \\
\text { Tímida } \\
\text { Tonta } \\
\text { Veleidosa } \\
\text { Voluble }\end{array}$ & $\begin{array}{l}\text { Afeminada } \\
\text { Amorosa } \\
\text { Casadera } \\
\text { Compasiva } \\
\text { Coqueta } \\
\text { Femenina } \\
\text { Fiel } \\
\text { Inocente } \\
\text { Insinuante } \\
\text { Monógama } \\
\text { Romántica } \\
\text { Seductora } \\
\text { Sensual }\end{array}$ \\
\hline
\end{tabular}

\section{Discusión}

Desde un punto de vista meramente descriptivo, al examinar la frecuencia de utilización de los vocablos seleccionados con las categorías más altas de exactitud con que cada término describe los comportamientos sexuales, el análisis de los datos permite afirmar, en primer lugar, que en adultos jóvenes de ambos sexos que cursan estudios terciarios y universitarios, y residen en un ámbito urbano en Buenos Aires y conurbano, existe una percepción bastante similar al calificar sus comportamientos sexuales: informan ser apasionados, espontáneos, tiernos, divertidos, cariñosos, heterosexuales y románticos. Cada grupo afirma, además, ser femenino o masculino, según su sexo. 
Sin embargo, las mujeres se describen más frecuentemente como fieles y monógamas, adjetivos que no se cuentan entre los más señalados por el grupo masculino. Los varones se califican como gentiles con alta frecuencia, cosa que no sucede en la muestra femenina.

Contemplando ahora la hipótesis planteada, se verifica la existencia de diferencias significativas en las respuestas de hombres y mujeres: ello implica que existen adjetivos que han sido utilizados predominantemente por cada grupo, a diferencia del otro grupo de comparación, que mayoritariamente no los ha empleado.

De todas maneras, no debe perderse de vista que las diferencias con significación estadística halladas entre ambos sexos se refieren a términos seleccionados, en líneas generales, con baja frecuencia. Sin embargo es digno de destacar que el grupo masculino elige atributos vinculados con ser adúltero, libertino, inmoral, obsceno, promiscuo, pervertido, ordinario. Estos calificativos no aparecen en las respuestas dadas por mujeres, quienes se identifican con las cualidades siguientes: casaderas, compasivas, coquetas, inocentes, seductoras, sensuales e insinuantes.

Considerando la comparación que se ha establecido con los resultados informados por Williams y Best (1994) sobre esterotipos de género, puede pensarse que, tomando como referencia la mirada del otro o las concepciones relacionadas con el self dialógico a las que hemos hecho referencia en la introducción de este trabajo, los comportamientos asociados con las identidades según género se configuran de manera diferente en mujeres y hombres. La mujer seduce e insinúa, busca una relación permanente. El varón se permite no ser fiel, ciertas obscenidades y perversiones, algo de inmoralidad. La imagen femenina corresponde a un personaje que busca ser conquistado agradando al otro; la masculina, a alguien dominante, activo - agresivo, que busca conquistar. De esas maneras diferenciadas quieren o desean ser mirados o reconocidos.

En una época en la que los mensajes culturales explícitos han borrado ciertas diferencias en los comportamientos sexuales de ambos sexos, la teoría se impone sobre una presunción ingenua, nacida del conocimiento vulgar o saber popular: aunque podría esperarse una menor diferenciación en las respuestas, en virtud del borramiento de las marcadas diferencias de los roles sexuales en nuestra sociedad actual, parecería que el uso diferencial de ciertos términos en las autodescripciones sexuales, estaría confirmando postulados de la teoría sociobiológica, que presenta un varón cuya promiscuidad aumenta las posibilidades de supervivencia de la especie, que se retira después de aparearse, y a una mujer que busca ese contacto, propendiendo, en última instancia, a conservar la protección del macho - para sí misma y para sus crías -. Cabe preguntarse, sin embargo, si estas 
respuestas se corresponden con los comportamientos efectivos que cada sexo ejecuta en torno a los rituales involucrados en la sexualidad, o solamente se trata de responder en el listado de adjetivos aquello que - suponen - se espera de ellos, por ser culturalmente aceptables tales descripciones.

Es claro que el presente estudio no se halla en condiciones de contrastar estas alternativas por la naturaleza de su diseño. ¿Estos estereotipos se ejecutan realmente, o simplemente, se enuncian? Es de esperar que futuras investigaciones profundicen en este análisis, considerando, además de otros posibles hábitats, grupos etarios y culturales, la evaluación de los comportamientos efectivos.

\section{Referencias Bibliográficas}

Archer, J. (1992). Childhood gender roles: social context and organization. En H. Mc Gurk (Ed.), Childhood social development: contemporary perspectives, (pp. 108 - 151). Hove: Elrlbaum.

Bakhtin, M. (1973) Problems of Dostoievsky poetics.Michigan: Arbis (original ruso, 1929).

Beck, U. y Beck-Gernshein, E. (2001) El normal caos del amor. Las nuevas formas de la relación amorosa. Barcelona: Paidós.

Bonder, G. (1985) Los estudios de la mujer y la crítica epistemológica a los paradigmas de las Ciencias Humanas. Buenos Aires: Dto. Publicaciones, Facultad de Psicología, Universidad de Buenos Aires.

Buss, D. M. (1987). Sex differences in human mate selection: an evolutionary perspective. En C. Crawford, M. Smith \& D. Krebs (Eds.), Sociobilogy and Psychology: ideas, issues and applications, (pp. 104 - 176). Hillsdale, N. J.: Erlbaum.

Buss, D. M. (1994). Mate preferences in 37 cultures. En W. J. Lonner y R. S. Malplass (Eds.), Psychology and culture (pp. 415 - 436). Boston: Allyn \& Bacon.

Buss, D. M., Abbott, M., Angleitner, A., Asherian, A., Biaggio, A. et al. (1990). International preferences in selecting mates. A study of 37 cultures. Journal of Cross - Cultural Psychology, 21(1), 5 - 47.

Casullo, M.M., Figueroa, N.B. y Aszkenazi, M. (1991). Teoría y técnicas de evaluación Psicológica. Buenos Aires: Psicoteca. 
De Beauvoir, S ( 1990). Del Amor. Madrid: Alianza.

Dorner, G. (1976) Hormones and brain differentiation. Amsterdam: Elsevier.

Durkin, K. (1995). Developmental social psychology: From infance to old age. Oxford: Blackwell.

Edley, N. y Wetherell, M. (1995). Men in perspective: practice, power and identity. New York: Prentice Hall/Harvester Wheatsheaf.

Fernández A. M. (1994). La mujer de la ilusión. Buenos Aires: Paidós.

Freud, S. (1996). Tres ensayos de teoría sexual, en Freud (1905) Obras completas. Buenos Aires: Amorrortu.

Freud, S. (1996). Organización genital infantil, en Freud (1923) Obras completas. Buenos Aires: Amorrortu.

Hermans, H.J. ( 2001). The Dialogical Self: Toward a theory of personal and cultural positioning. Culture \& Psychology, 7(3), 243 - 281.

Hermans, H.J., Kempen, H.J. y Van Loon, R.J. (1992). The Dialogical self: Beyond individualism and rationalism. American Psychologist, 47, 23 - 33.

Hofstede, G.(1999). Culturas y Organizaciones. El software mental. Madrid: Alianza.

James, W. ( 1890). The principles of Psychology. Vol.1. New York: Henry Holt.

Jodelet D. (1986). La representación social: fenómenos, concepto y teoría. En S. Moscovici (Ed.) Psicología Social. Barcelona: Paidós.

Jost, A. (1970). Hormonal factors in sexual differentiation. Philosofical Transactions of the Royal Society of London, B259, 119 - 130.

Lacan, J. (2000). Seminario XX. (1973). Aún. Buenos Aires: Paidós.

Laplanche, J. y Pontalis J. B. (1981), Diccionario de Psicoanálisis. Madrid: Labor. Lipovetsky, G. (1999). La tercera mujer. Barcelona: Anagrama.

Maccoby, E. E. \& Jacklin, C. N. (1994). The psychology of sex differences. 
Stanford, California: Stanford University Press.

Meler, I. (1994). Parejas de la transición: entre la psicopatología y la respuesta creativa. Actualidad Psicológica., ( 8 ), 7- 12.

Money, J. y Ehrhardt, A. A. (1972). Man and woman, boy and girl. Baltimore: Johns Hopkins University Press.

Ornstein, R. (1986). The psychology of consciousness. Harmondsworth: Penguin.

Schmitt, D. P. \& Buss, D. M. (2001). Human Mate Poaching: Tactics and Tempatations for Infiltrating Existing Mateships. Journal of Personality and Social Psychology, 80(6), 894 - 917.

Townsend, J. (1998). Lo que quieren las mujeres, lo que quieren los hombres. México: Oxford.

Williams, J. E. y Best, D. L. (1994). Cross - cultural views of women and men. En W. J. Lonner y R. S. Malplass (Eds.), Psychology and culture (pp. 212 - 326). Boston: Allyn y Bacon. 